\title{
Polarization Multiplexed Interrogation Technique for FBG Sensor Array
}

\author{
Debabrata SIKDAR, Vinita TIWARI, Anupam SONI, Ritesh JAISWAL*, \\ and Surekha BHANOT
}

Laboratory of Optical Fiber Communications, Birla Institute of Technology and Science Pilani, Rajasthan 333031, India

*Corresponding author: Ritesh JAISWAL_ E-mail: riteshjais93@gmail.com

\begin{abstract}
This paper proposes a polarization multiplexed interrogation technique for fiber Bragg grating (FBG) sensor array. The novelty of the proposed model is its ability to reduce interference and cross talk, thus allowing larger number of FBG sensors to be interrogated in an array. The calibration technique has been illustrated in this work for the FBG sensor array, where data from each sensor are linearly polarized and multiplexed before co-propagation, to find out the tapping points that enable identification of each sensor data uniquely. Simulation has been carried out for odd number and even number of sensors in an array. Even with interfering input, this proposed scheme can interrogate and distinctively identify each sensor data using appropriate tuning of polarization-splitter, polarization-rotator, and polarization-attenuator at the detector end during the calibration process. The significance of the proposed method is its compact size, which makes this calibration system ready to be deployed in real-time sensing applications and data acquisition from the FBG sensor array.
\end{abstract}

Keywords: FBG, wavelength division multiplexing (WDM), time division multiplexing (TDM), spatial division multiplexing (SDM), fiber-optic sensors

Citation: Debabrata SIKDAR, Vinita TIWARI, Anupam SONI, Ritesh JAISWAL, and Surekha BHANOT, "Polarization Multiplexed Interrogation Technique for FBG Sensor Array ," Photonic Sensors, 2015, 5(3): 193-201.

\section{Introduction}

Over the last few decades, the fiber optic technology has seen a tremendous growth both in the fields of telecommunications and sensing [1-4]. Besides numerous advantages of the fiber-optic technology in telecommunication application [5-8], fiber-optic sensors especially FBG sensors have gained popularity in fiber optic sensing applications because of their simplicity, low cost, minimal electromagnetic interference, wide dynamic range, negligible loading effect, and relatively long distance communication. Hence, the fiber-optic sensing technology is a potential alternative to the traditional sensors for acceleration, rotation, electric and magnetic field measurement, temperature, pressure, displacement, acoustics, vibration, linear and angular position, stress, strain, viscosity, humidity chemical compositions, and many other sensing applications [1-3, 9-14]. Compared with other implementations of fiber-optic sensors, FBG sensors offer a distinguishing advantage over others through the insensitivity of absolute measurement to the source fluctuations as the detection is purely based on the wavelength shift introduced by the measurand. Thus, FBG sensors are very suitable for sensing and data acquisition, where sensor arrays

Received: 4 December 2014 / Revised version: 26 April 2015

(C) The Author(s) 2015. This article is published with open access at Springerlink.com DOI: $10.1007 / \mathrm{s} 13320-015-0235-2$

Article type: Regular 
can be multiplexed using similar techniques that have been applied for fiber-optic sensors like wavelength-division multiplexing (WDM), spatial-division-multiplexing (SDM), and time-division-multiplexing (TDM) as they can be directly implemented in the fiber without changing the diameter of the fiber. This feature makes FBG sensors suitable for a wide range of applications [15, 17-19].

\section{Theory of FBG sensors}

When a broad-spectrum light beam is incident on an FBG, reflections from each segment of alternating refractive index interfere constructively only for a specific wavelength of light, called the Bragg wavelength, $\lambda_{B}$. This effectively causes the FBG to reflect a specific frequency of light while transmitting all others. The Bragg wavelength $\lambda_{B}$ is a function of the spacing between the gratings $(\Lambda)$ and effective refractive index $\left(n_{e}\right)$ of the fiber core. The equation of the Bragg wavelength is

$$
\lambda_{B}=2 n_{e} \Lambda .
$$

Any changes in external stimuli viz. temperature, strain, vibration etc. will affect the effective refractive index $n_{e}$ and grating period ( $\Lambda$ ) of an FBG, which results in a shift in the reflected wavelength. The change in the wavelength of an FBG due to both strain and temperature is represented as

$$
\frac{\Delta \lambda_{B}}{\lambda_{B}}=\left(1-p_{e}\right) \varepsilon+\left(\alpha_{A}+\alpha_{n}\right) \Delta T
$$

where $\Delta \lambda_{B}$ is the wavelength shift, and $\lambda_{B}$ is the initial wavelength. In (2), the first term describes the impact of strain on the wavelength shift, where $p_{e}$ is the strain-optic coefficient $(\sim 1 \mathrm{pm} / \mu \varepsilon)$, and $\varepsilon$ is the strain experienced by the grating. The second term describes the impact of temperature on the wavelength shift, where $\alpha_{\Lambda}$ is the thermal expansion coefficient, and $\alpha_{n}$ is the thermo-optic coefficient. The coefficient $\alpha_{n}$ describes the change in the refractive index, while $\alpha_{\Lambda}$ describes the expansion of the grating $(\sim 10 \mathrm{pm} / \mathrm{K})$, both due to temperature. Normally, the FBG will respond to both strain and temperature, so we need to account for both effects and distinguish between the two. For sensing temperature, the fiber Bragg grating must remain unstrained to make strain not affect the value of temperature which we need to calculate. This can be achieved by using packaged FBG temperature sensors to ensure the FBG inside the package is not subjected to any bending, tension, compression or torsion forces. The expansion coefficient $\alpha_{\Lambda}$ of glass is practically negligible, thus the changes in the reflected wavelength due to temperature can be principally described by the change in the refractive index, $\alpha_{n}$ of the fiber. For measurement of strain, the changes in the reflected wavelength primarily describe the change in the expansion coefficient $\alpha_{\Lambda}$ of the glass optical fiber [9, 10, 18, and 19]. Hence, the essence of FBG based sensing is to accurately identify $\Delta \lambda_{B}$.

FBG sensors have found the application in sensing diverse external environmental stimuli, and these sensors are commonly deployed now-a-days for various applications viz. civil structural health monitoring and diverse scientific research $[1-4$, 9-11].

\section{Existing interrogation techniques for FBG sensor arrays and their major limitations}

Accurate measurement of the FBG wavelength shift induced by the measurand is crucial for achieving good sensor performance. The general requirement of an ideal interrogation method is to have high resolution, ability to support multiplexing, be robust and cost effective. Various interrogating techniques are being used for FBG sensors but the commercial interrogators for FBG sensors can be broadly classified as TDM, WDM, SDM, and their various combinations for the improved performance $[15,17-22]$. TDM systems uses a pulse from a broadband light source, and all sensors are nominally written at the same wavelength with low reflectivity, thus allowing some light to pass down 
the fiber to illuminate the sensors downstream. The TDM differentiates among different gratings by the time taken for their return signal to reach the detector. TDM sensors are lightweight and rugged and also offer faster sampling speed at comparatively low cost. The main problem with the TDM system is that the sensors must be placed sufficiently far apart because the pulse returning from the adjacent sensors must be able to reach and get detected separately. In WDM systems, different sensors have the nominal central wavelength, and other sensors are separated by a few nanometers. Each sensor is tracked simultaneously as its central wavelength changes due to temperature, strain or pressure. WDM interrogation is available in two topologies i.e., series and parallel. The parallel approach is easier to implement but the series topology allows the optical power from the sensing FBG array to be used much more efficiently than parallel topology. The WDM method offers high resolution, accuracy, stability, and flexibility with moderate numbers of sensors and relatively low sampling rates. The WDM sensor technology is well-developed but sensors come with large size and relatively high cost. The TDM/WDM topology is able to address the large number of FBG sensors and is one of the most promising techniques for quasi-distributed measurement [20-24]. The drawback of this type of implementation is the limited scanning frequency, which would limit the response of the system to dynamic signals and transients, and limited spatial resolution. FBGs can be spaced no closer than 1 meter even for the best TDM systems. The SDM topology is the choice of preference in aerospace and security applications where point measurement is required because in this scheme, FBG sensors in a network are operated independently, and the sensor can be interchangeable and replaceable without any substantial recalibration in the event of damage.

Major limitations in interrogating FBG sensor arrays are the cross-talk, spectral shadowing, and interference. For all the interrogation approaches discussed above, some crosstalk between adjacent sensors seems to be unavoidable. The use of a serial array of FBG sensors with the same central wavelength results in the crosstalk between sensors. The amount of light reflected by the FBG sensors located nearest to the source will affect the amount of the optical power reaching and being returned from gratings further from the source. The lower the peak reflectivity of the FBGs is, the smaller the effect is. Another source of the crosstalk in a TDM serial array of identical FBG sensors arises from multiple reflections between FBGs. This can lead to pulses arriving simultaneously at the detector having undergone a direct reflection from a sensor element and also having experienced a number of multiple reflection paths between FBGs. The effect is proportional to the grating's reflectivity and can be minimized using low reflectivity gratings $(<5 \%)$. Thus the number of grating arrays that can be interrogated under a given signal-to-noise ratio will depend on the amount of the crosstalk. Thus for interrogating the large array of grating, pairing of the low-reflective and high sensitivity grating is essential.

Spectral shadowing is the distortion introduced in the reflected spectrum of a downstream grating resulting from the double pass of the incoming light through an upstream grating. Shadowing effect can be eliminated by staggering the grating array to avoid spectral overlapping. Time overlap can be reduced by increasing the time interval between gratings using passive delay lines. Interference could occur when the reflected optical signal that one would expect from a single isolated fiber-optic FBG sensor is somehow altered by the existence of other gratings [16-18].

\section{Proposed method of polarization multiplexed interrogation}

This paper deals with the concept of polarization 
division multiplexing as a method for interrogation of the FBG sensor array with the minimum interference and crosstalk issues. The motivation behind this concept to be used in FBG sensor interrogation lies in polarization division multiplexing (PDM) implementation in the high speed optical network [19, 20]. Here, each FBG sensor needs to have a polarizer with the known predetermined angle of polarization. The reflected lights from different sensors which are aligned to the unique angles of polarization co-propagate through the fiber with the minimum interference and crosstalk. The appropriate use of polarization rotator and polarization attenuator can separate out the reflected light from a particular sensor and measure the change in the physical parameter in terms of the change in the reflected wavelength for a designated FBG sensor.

\section{Simulation model for FBG sensor array calibration and interrogation}

The system model used in this work has been developed using commercial simulation software OptiSystem. The model uses a broadband optical source as a transmitter which has been generated using the nonlinear phenomenon of cross phase modulation (XPM). Two continuous-wave laser sources with different wavelengths co-propagate in a small segment of the optical fiber with considerably high launch power, and the nonlinear phase fluctuations eventually lead to substantial spectral broadening. This broad optical spectrum acts as a broadband optical source after the desired frequency band is filtered out using a Gaussian bandpass filter. The components preceding the first sensor is the simulated model of the broadband optical source generated using the concept of fiber nonlinearity. To implement the concept of PDM in FBG sensor arrays, two different cases consisting of a) odd and b) even number sensors in an array have been considered. In the simulation model, the reflectivity of FBG sensors is considered to be $20 \%$. The calibration done at the time of installation of such sensor array is the essence of this concept. In this work, two different cases of " 3 " (odd) and " 4 " (even) sensors in an array have been simulated to demonstrate the practical realization of the concept which can be further extended for a larger number of sensors in FBG sensor array.

\subsection{Array of odd number of FBG sensors}

To demonstrate the calibration and interrogation procedure for the polarization multiplexed FBG sensor array, a sensor array comprising of the odd number of sensors has been considered for simulation in this case.

\subsubsection{System model for calibration and interrogation}

Figure 1 shows the simulation setup for calibration of 3 sensors to simulate the case for the odd number of FBG sensors in a sensor array. S1, S2, and $\mathrm{S} 3$ are the three FBG sensors connected serially. Each sensor is made to reflect a predetermined wavelength, and the reflected pulse also undergoes a polarizer that introduces a known angle of polarization.

The reflected lights from all three sensors co-propagate while travelling back towards the detector located at the transmitter end itself. The co-propagation of reflected lights from the sensors back towards the transmitting end, at which the detector is located, is simulated using the fiber segments named Optical Fiber_32 for back propagating light where Optical Fiber_23 carries the light in the forward direction from FBG2 to FBG3 in Fig. 1. The reflected signals are made to differ by $5^{\circ}$ of polarization with their neighboring polarized pulses. As shown in Fig. 1, the appropriate polarization splitters, polarization rotators, and polarization attenuators are used to finally figure out three tapping points where the reflected wavelengths from three sensors can be identified. It can be seen that the oscilloscope at tapping point $\mathrm{T} 1$ identifies the wavelengths corresponding to $\mathrm{S} 1$ and $\mathrm{S} 3$ 
whereas the oscilloscope at tapping point $\mathrm{T} 2$ identifies the wavelengths corresponding to $\mathrm{S} 1$ and $\mathrm{S} 2$. The signal from tapping point $\mathrm{T} 1$ has further passed through a polarization rotator and a polarization attenuator which blocks S1 and allows S3 at tapping point T3.

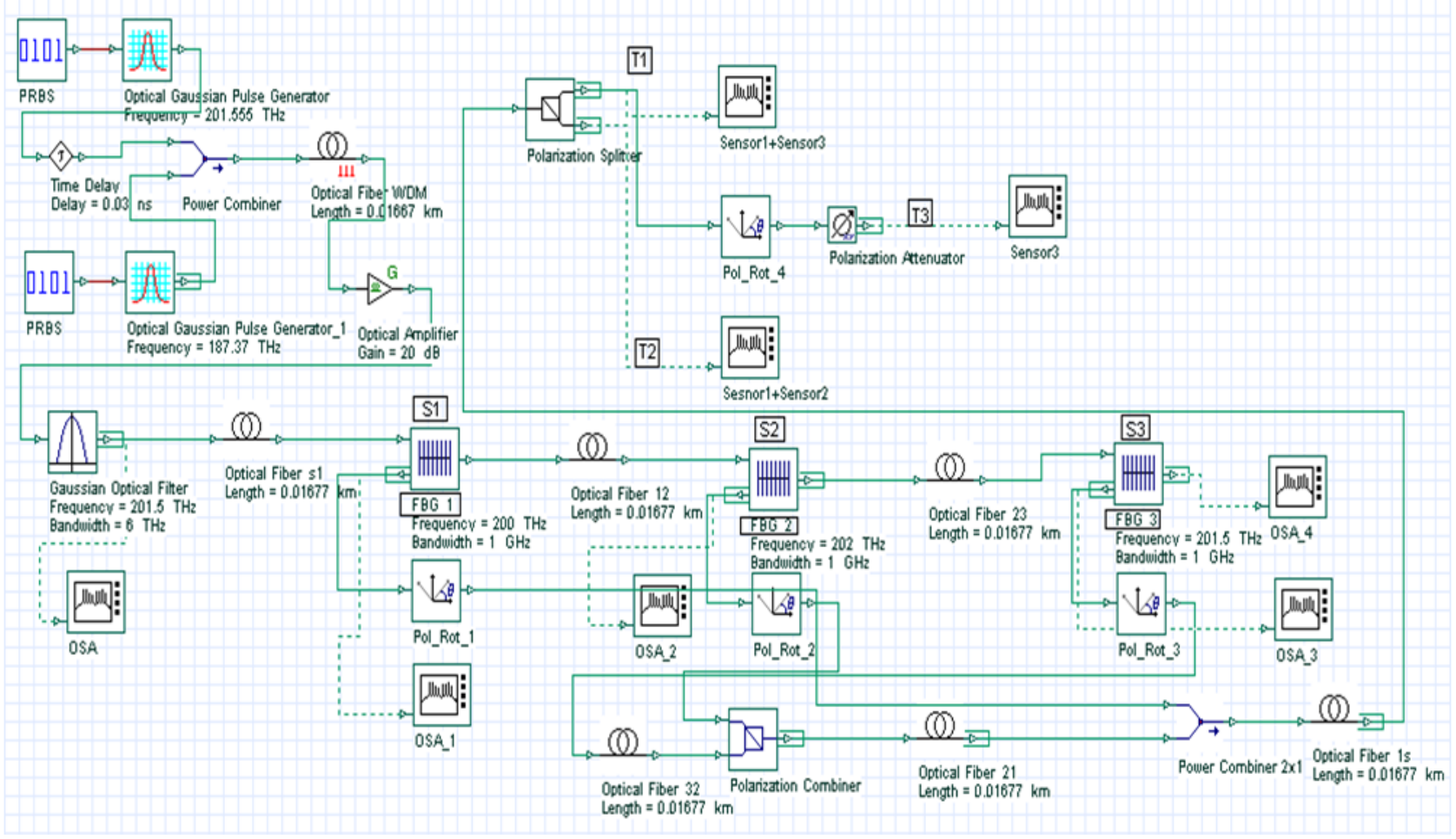

Fig. 1 Simulation setup for calibration and interrogation of FBG sensor array of 3 sensors.

Thus from T3 point, S3 can be uniquely identified which allows S1 to be identified from tapping point $\mathrm{T} 1$. Identification of $\mathrm{S} 1$ helps identifying $\mathrm{S} 2$ uniquely from tapping point $\mathrm{T} 2$. Thus this arrangement can uniquely identify all three polarization multiplexed signals coming from the sensors positioned at S1, S2, and S3. In the simulation model for calibration purpose of the system model parameters, S1 FBG reflects back $200 \mathrm{THz}$ frequency while S2 and S3 are assumed to reflect back $202 \mathrm{THz}$ and $201.5 \mathrm{THz}$, respectively. Reflected light from $\mathrm{S} 1$ has been polarized to $5^{\circ}$ while the reflected lights from S2 and S3 undergo polarization rotation of $10^{\circ}$ and $15^{\circ}$, respectively. While interrogating these 3 sensors, the oscilloscopes at tapping point $\mathrm{T} 2$, T1, and $\mathrm{T} 3$ identify each sensor data uniquely.

\subsubsection{Simulation result for 3-sensor array interrogation}

It can be seen from Fig. 2(c) that S3 reflects back 201.5 THz. Then, from Fig. 2(b), S1 can be identified to reflect back $200 \mathrm{THz}$. Thus signals from S1 and S3 can be identified from tapping positions T1 and T3. Furthermore, the oscilloscope at tapping point $\mathrm{T} 2$ provides information about $\mathrm{S} 2$ which is reflected at $202 \mathrm{THz}$. Thus for any odd number FBG sensor in an array, data from all the sensors can be uniquely identified in this scheme which is least affected by the interference and crosstalk.

\subsection{Array of even number of FBG sensors}

To demonstrate the calibration and interrogation procedure for the polarization multiplexed FBG sensor array, a sensor array having even number of sensors has been considered for simulation in this case. 


\subsubsection{System model for calibration and interrogation}

This scheme has been further deployed to interrogate $4 \mathrm{FBG}$ sensors (even number sensor in the array) as shown in Fig. 3. Here, we have considered S1, S2, S3, and S4 to reflect back $200 \mathrm{THz}, 202 \mathrm{THz}, 201.5 \mathrm{THz}$, and $202 \mathrm{THz}$, and the reflected light from these sensors are rotated by the polarization angle of $5^{\circ}, 10^{\circ}, 15^{\circ}$, and $20^{\circ}$, respectively. S2 and S4 are considered to reflect back the same frequency so that we can investigate the effect of interference.

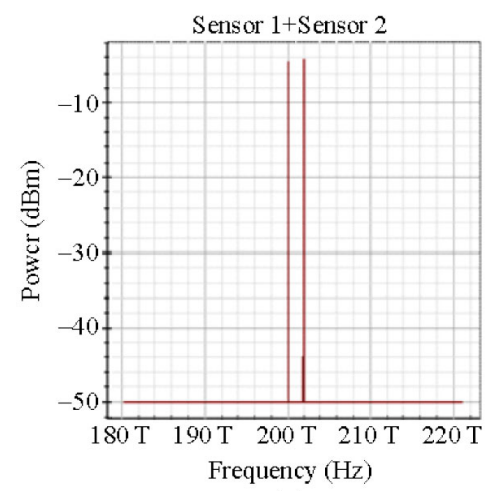

(a)

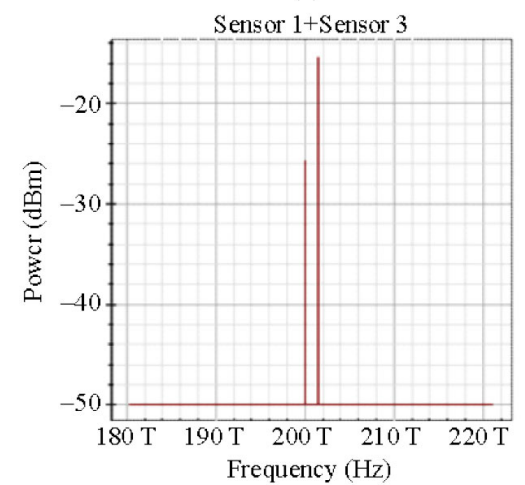

(b)

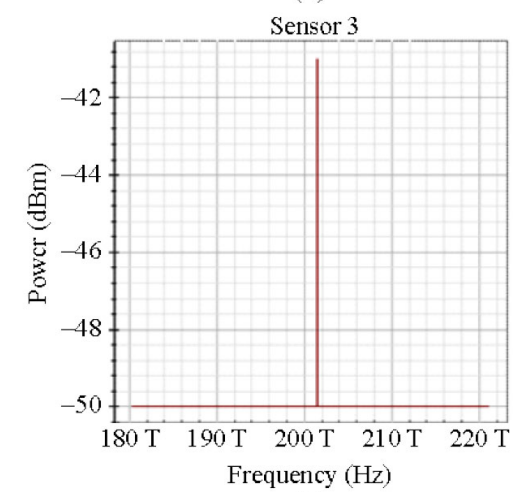

(c)

Fig. 2 Simulation result of interrogating a 3-sensor array.
This system model simply contains an additional FBG sensor in the array. The method of simulating optical broadband source, polarization multiplexing of co-propagating back-side reflected light from the sensor remains the same as discussed in Section 4.1.1. Thus, it can be realized that this scheme implements the identification of tapping points in a top-down approach while the interrogation process for reading individual sensor data is performed in a bottom-up approach. The essence of this scheme is to calibrate the polarization-splitter, rotator, and attenuator and identify the tapping points which are done in a top-down approach for known values of sensor data. When polarization multiplexed signals $(\mathrm{S} 1+\mathrm{S} 2+\mathrm{S} 3+\mathrm{S} 4)$ co-propagating in the fiber reach the detector, it is split into 2 sub-streams of $(\mathrm{S} 1+\mathrm{S} 2+\mathrm{S} 3)$ and $(\mathrm{S} 1+\mathrm{S} 2+\mathrm{S} 4)$ using the polarization splitter. $(\mathrm{S} 1+\mathrm{S} 2+\mathrm{S} 3)$ has been further processed by attenuating $\mathrm{S} 1$, thus extracting $(\mathrm{S} 2+\mathrm{S} 3)$ which then passes through another polarization attenuator that removes $\mathrm{S} 3$ and uniquely identifies $\mathrm{S} 2$. The tapping points for $(\mathrm{S} 1+\mathrm{S} 2+\mathrm{S} 3),(\mathrm{S} 1+\mathrm{S} 2+\mathrm{S} 4),(\mathrm{S} 2+\mathrm{S} 3)$, and (S2) are shown as T1, T2, T3, and T4 in Fig. 3.

\subsubsection{Simulation result for 4-sensor array interrogation}

It can be seen from Fig. 3 that here we have 4 tapping points to uniquely identify data from all four sensors. The oscilloscope readings from the four tapping points are shown in Figs. 4(a), 4(b), 4(c), and 4(d). Identification of sensor data can be started from Fig. 4(d) which displays only S2 data i.e., 202 THz. Then Fig. 4(c) informs about data from S3 i.e., 201.5 THz. When S2 and S3 are identified, Fig. 4(b) helps in identifying S1 i.e., $200 \mathrm{THz}$. Now as S1 and S2 are known, Fig. 4(a) uniquely identifies data from S4. In the subplot, only two frequencies are displayed which are $200 \mathrm{THz}$ and $202 \mathrm{THz}$ of which $200 \mathrm{THz}$ is S1 data leaving S2 and S4 to have a reflected wavelength at $202 \mathrm{THz}$. Thus sensors at different locations having the same data can also be identified and thus minimizes the limitations found 
in existing interrogation techniques.

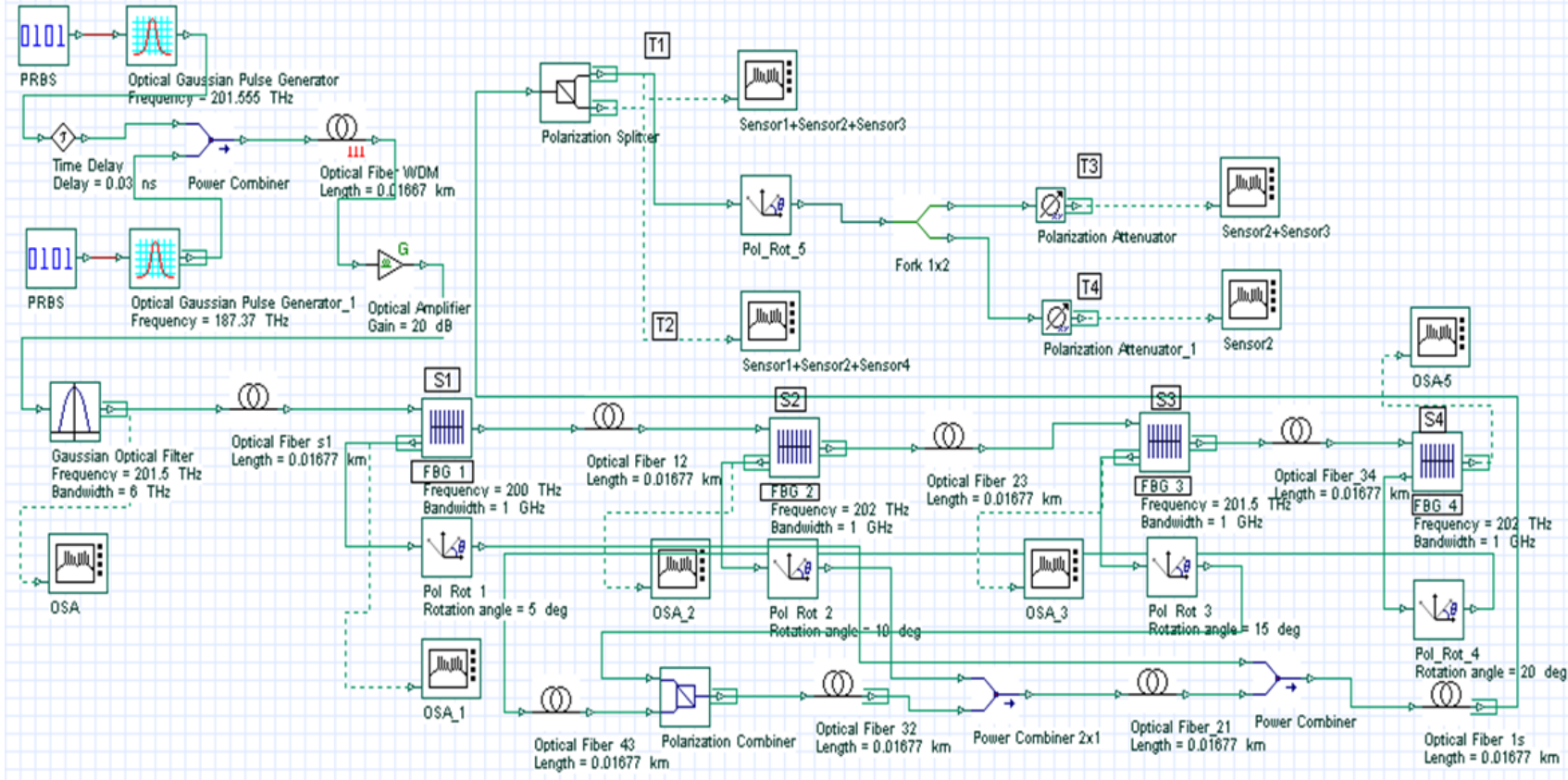

Fig. 3 Simulation setup for calibration and interrogation of FBG sensor array of 4 sensors.

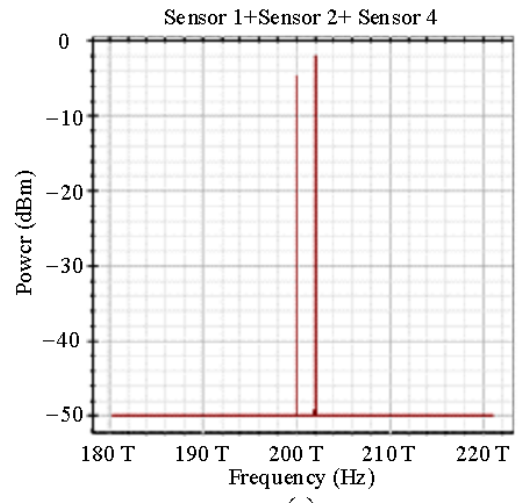

(a)

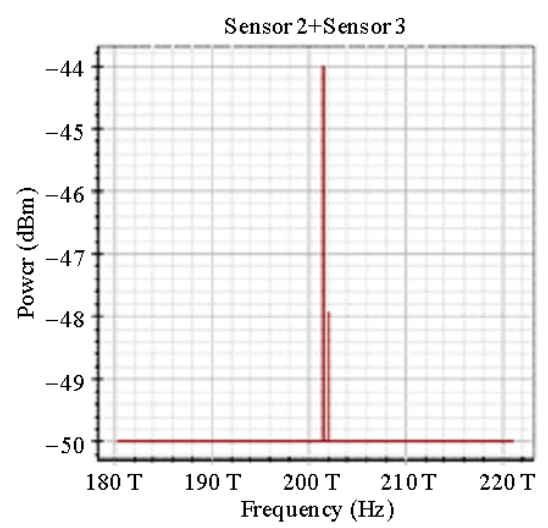

(c)

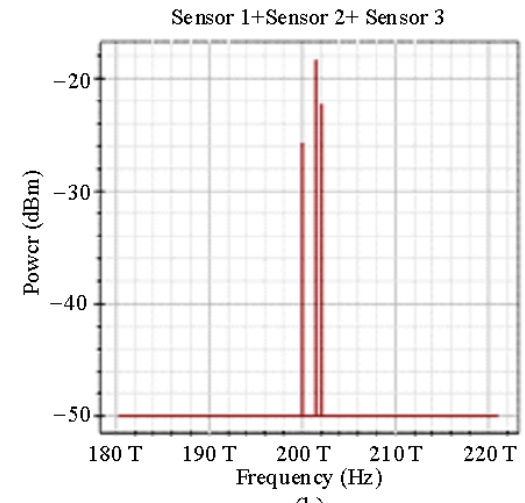

(b)

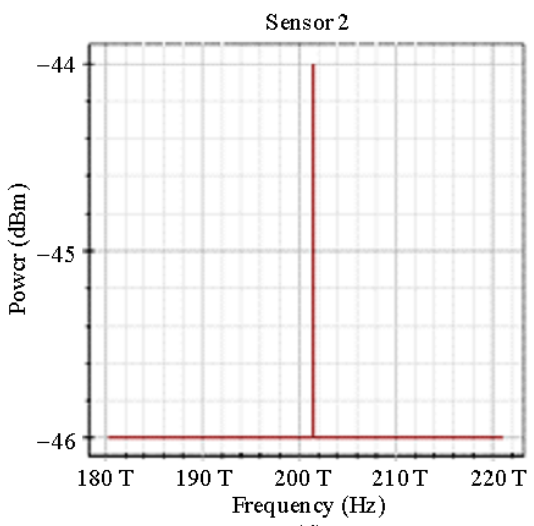

(d)

Fig. 4 Simulation result of interrogating 4-sensor array. 


\subsection{Array of $\boldsymbol{n}$ number of FBG sensors}

From the analyses presented in Sections 4.1 and 4.2, the proposed concept of polarization multiplexed interrogation can be extended in a generalized way for analyzing a sensor array consisting of $n$ FBG sensors. The system needs to be calibrated for known frequencies of all measured i.e., $n$ FBG sensors' reflected wavelength where $n$ tapping points as discussed in Section 5 need to be identified distinctively. The process starts by splitting the combined backward propagating reflected lights coming from $n$ sensors into two streams containing data for $n 1$ sensors.

Then by using the polarization rotator and attenuator, one needs to keep on suppressing one sensor data and identifying the corresponding tapping point unless the single FBG sensor data are identified uniquely. This top-down approach for calibration is necessary before the identification of tapping points by using the bottom-up approach sensor data information can be extracted.

\section{Conclusions}

This paper presents the simulative concept for implementing the polarization multiplexing technique in the interrogation of the FBG sensor array. This proposed technique needs calibration as demonstrated in the paper for the FBG sensor array consisting of odd or even number of sensors. The sensor array calibration is performed, and the tapping points for different sensor data combinations are identified which leads to unique identification of each sensor data. After calibration, the system is ready to be deployed for real-time measurement of physical parameters. The main advantage of this technique is its small size even if the number of sensors in an array is large as the sensors in an array are separated from each other by small angles $\left(5^{\circ}\right)$. The technique developed has also the capability of rejecting interference and crosstalk from other sensors in the sensor array resulting in a more accurate measurement even if sensors at different locations have the same data, the sensors can also be properly identified, thus can be useful for the applications such as oil \& gas industry where thousands of kilometers of fiber cables are laid to monitor the production of the field.

Open Access This article is distributed under the terms of the Creative Commons Attribution License which permits any use, distribution, and reproduction in any medium, provided the original author(s) and source are credited.

\section{References}

[1] A. D. Kersey, M. A. Davis, H. J. Patrick, M. LeBlanc, K. P. Koo, C. G. Askins, et al., "Fiber grating sensors," Journal of Lightwave Technology, 1997, 15(8): 1442-1463.

[2] Y. J. Rao, "In-fiber Bragg grating sensor," Measurement Science and Technology, 1997, 8(4): 355-375.

[3] M. G. Xu, H. Geiger, and J. P. Dakin, "Modeling and performance analysis of a fiber Bragg grating interrogation system using an acousto-optic tunable filter," Journal of Lightwave Technology, 1996, 14(3): 391-396.

[4] A. Othonos and K. Kalli, Fiber Bragg gratings: fundamentals and applications in telecommunications and sensing. Boston, MA: Artech, 1999.

[5] D. Sikdar, V. Tiwari, Y. Saha, and V. K. Chaubey, "Investigation of modulator chirp and extinction ratio in different RZ-and NRZ duobinary transmitter modules for performance optimization," Optik International Journal for Light and Electron Optics, 2013, 124 (13): 1411-1414.

[6] D. Sikdar, V. Tiwari, and V. K. Chaubey, "Investigation of RZ and NRZ pulse shape for optimum Duobinary transmission at 40 Gbps," Optik - International Journal for Light and Electron Optics, 2013, 124 (12): 1148-1151.

[7] V. Tiwari, D. Sikdar, and V. K. Chaubey, "Performance optimization of RZ-DQPSK modulation scheme for dispersion compensated optical link," Optik - International Journal for Light and Electron Optics, 2013, 124 (17): 2593-2596.

[8] D. Sikdar, V. Tiwari, and V. K. Chaubey, "Optimum dispersion map profile for a stable DM soliton system," Journal of Modern Optics, 2012, 59(16): 1396-1405.

[9] J. Hu, Z. Chen, and C. Yu, "150-km long distance FBG temperature and vibration sensor system based 
on stimulated Raman amplification," Journal of Lightwave Technology, 2012, 30(8): 1237-1243.

[10] C. H. Yeh, C. W. Chow, C. H. Wang, F. Y. Shih, Y. F. $\mathrm{Wu}$, and S. Chi, "A simple self-restored fiber Bragg grating(FBG)-based passive sensing network," Measurement Science and Technology, 2009, 20(4): 043001

[11] P. C. Peng, H. Y. Tseng, and S. Chi, "Fiber-ring laser-based fiber grating sensor system using self-Healing ring architecture," Microwave and Optical Technology Letters, 2002, 35(6): 441-444.

[12] B. Zhang and M. Kahrizi, "High-temperature resistance fiber Bragg grating temperature sensor fabrication," IEEE Sensors Journal, 2007, 7(4): 586-591.

[13] L. Jin, W. Zhang, H. Zhang, B. Liu, J. Zhao, Q. Tu, et al., "An embedded FBG sensor for simultaneous measurement of stress and temperature," IEEE Photonics Technology Letter, 2006, 18(1): 154-156.

[14] Y. Zhao, H. W. Zhao, X. Y. Zhang, Q. Y. Meng, and B. Yuan, "A novel double-arched-beam-based fiber Bragg grating sensor for displacement measurement," IEEE Photonics Technology Letter, 2008, 20(15): 1296-1298.

[15] C. H. Yeh, M. C. Lin, C. C. Lee, and S. Chi, "Fiber Bragg grating-based multiplexed sensing system employing fiber laser scheme with semiconductor optical amplifier," Japanese Journal Applied Physics, 2005, 44(1): 6590-6592.

[16] C. H. Yeh and S. Chi, "Fiber-fault monitoring technique for passive optical networks based on fiber Bragg gratings and semiconductor optical amplifier," Optics Communications, 2006, 257(2): 306-310.

[17] S. W. James, M. L. Dockney, and R. P. Tatam, "Simultaneous independent temperature and strain measurement using in-fiber Bragg grating sensors," Electronics Letters, 1996, 32(12): 1133-1134.

[18] H. J. Patrick, G. M. Williams, A. D. Kersey, J. R. Pedrazzani, and A. M. Vengsarkar, "Hybrid fiber Bragg grating/long period fiber grating sensor for strain and/or temperature discrimination," IEEE Photonics Technology Letters, 1996, 8(9): 1223-1225.

[19] Y. Miao, B. Liu, and Q. Zhao, "Simultaneous measurement of strain and temperature using single fiber Bragg grating," Electronics Letters, 2008, 44(21): 1242-1243.

[20] W. H. Chung, H. Y. Tam, P. K. A. Wai, and A. Khandelwal, "Time- and wavelength-division multiplexing of FBG sensors using a semiconductor optical amplifier in ring cavity configuration," IEEE Photonics Technology Letters, 2005, 17(12): 2709-2711.

[21] D. C. C. Norman, D. J. Webb, and R. D. Pechstedt, "Extended range interrogation of wavelength division multiplexed Fibre Bragg grating sensors using arrayed waveguide grating," Electronics Letters, 2003, 39(24): 1714-1716.

[22] Y. Sano and T. Yoshino, "Fast optical wavelength interrogator employing arrayed waveguide grating for distributed fiber Bragg grating sensors," Journal of Light Wave Technology, 2003, 21(1): 132-139.

[23] N. Cvijetic, D. Qian, J. Yu, Y. Huang, and T. Wang, "Polarization-multiplexed optical wireless transmission with coherent detection," Journal of Light Wave Technology, 2010, 28(8): 1218-1227.

[24] X. Xie, F. Yaman, X. Zhou, and G. Li, "Polarization demultiplexing by independent component analysis," IEEE Photonics Technology Letters, 2010, 22(11): 805-807. 